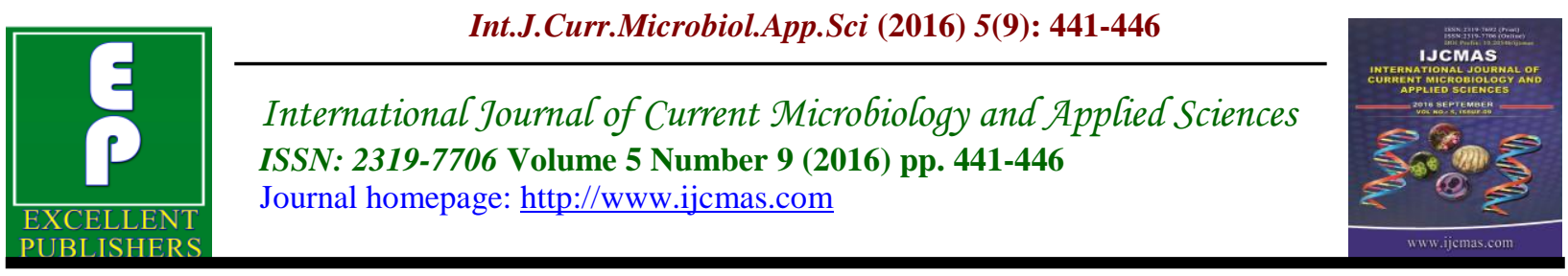

Original Research Article

http://dx.doi.org/10.20546/ijcmas.2016.509.047

\title{
A Study on Bacterial Flora on the Finger printing Surface of the Biometric Devices at a Tertiary Care Hospital
}

\author{
S. Nirupa*, V. Gayathri and Priyadarshini Shanmugam \\ Chettinad Hospital \& Research Institute, OMR, Rajiv Gandhi Salai, \\ Kelambakkam, Tamil Nadu, India \\ *Corresponding author
}

\begin{tabular}{l} 
Ke y w o r d s \\
Biometric Devices, \\
Bacterial Flora, \\
Finger printing \\
Surface, \\
indirect \\
transmission. \\
\hline Article Info \\
\hline Accepted: \\
15 August 2016 \\
Available Online: \\
10 September 2016
\end{tabular}

\section{A B S T R A C T}

Biometric finger printing devices are being more commonly used in both the public and private sectors to record attendance. This system involves the physical contact between the skin and the surface of the device. When successive persons align their fingers on the device there is the chance of the indirect transmission of the microorganisms from one person to another. Many studies have been carried out on the health care workers and also in the community settings as they are more prone to transfer the pathogenic microorganisms. In this study the microorganisms in the fingerprint devices in a tertiary care hospital were studied. This study aims to assess the risk of transmission of pathogenic bacteria through fingerprinting devices by isolating the bacterial flora which may be present in the Biometric fingerprinting device. The swabs were collected from the surface of the biometric fingerprinting device. The collected swabs were inoculated in the Blood agar plate and Mac Conkey agar plate and incubated overnight at $37^{\circ} \mathrm{C}$ for the isolation of bacteria. The identification of the bacterial isolates was done by performing Gram staining and biochemical reactions. The antibiotic sensitivity testing was done for the isolated pathogen. Of the total of 84 samples that were collected from the surface of the biometric fingerprinting devices, 39 (46\%) samples were culture positive. Among the culture positives, Coagulase negative Staphylococcus species (CONS) was the commonest organism to be isolated 19 (49\%), followed by Gram positive bacilli 17 (44\%) and Gram negative bacilli of 3(7\%). The 3 Gram negative bacilli isolated were Enterobacter spp., Acinetobacter spp and Aeromonas spp.

\section{Introduction}

Biometric fingerprinting devices are nowadays very commonly used both in the public and private sectors. The biometric fingerprinting device recognition is by the physical contact between the skin and the surface of the device. Many people successively align their fingers on the same surface. Hence this action may lead to the transmission of the microorganisms from these environmental devices to human beings. This can pose a risk of transmission of infection when it comes to devices used by health care workers whose hands are more prone to carriage of microorganisms including pathogens (Scientific Advisory Board of the International Scientific Forum 
on Home Hygiene, 2006; Centers for Disease Control and Prevention, 2002; Centers for Disease Control and Prevention, 2003). Many studies have been carried out on objects such as thermometers, stethoscopes, keyboard covers, ball pens, mobile phones, etc. (French et al., 1998; Neely et al., 1999) Microorganisms may be present in visibly clean hands. Most of the bacteria remain viable in the hands for about 30 minutes and they survive for a longer time on inanimate objects. The survival of the microorganism depends on the temperature, humidity and the presence of the organic matter (Carter, 2005; Kramer et al., 2006). This study was conducted to find out the microbial flora on the surface of the biometric fingerprinting device and thereby assess the risk of transmission of pathogenic bacteria through fingerprinting devices.

\section{Materials and Methods}

This study was carried out in Chettinad Hospital \& Research Institute, OMR, Rajiv Gandhi Salai, Kelambakkam, Tamil Nadu, India a tertiary care hospital. All biometric devices used by health care staff, administration staff, medical students, hostel inmates were included in this study. These devices were used to record attendance of students, staff and to offer access to certain areas like girls' hostel, boys' hostel, academic block, etc. There were 84 of them. Swabs were moistened with sterile saline before swabbing the surface of the biometric fingerprinting device. The collected swabs were inoculated in blood agar and Mac Conkey agar plate for the isolation of the bacteria if present. If any growth occurred, then identification of the bacteria was done by performing Gram staining and biochemical reactions. Antibiotic sensitivity testing was done for the isolated organisms according to the CLSI guidelines.

\section{Results and Discussion}

The total number of swabs collected from the surface of the biometric fingerprinting device was 84 . Based on the people using the biometric fingerprinting devices, the samples were grouped into four. Those devices in the college block (36) were used to record attendance of students, doctors and administrative staff, while those in common areas (16) are used by students and healthcare workers for the purpose of access to certain areas. The devices in the hospital block (24) were used to record attendance of students and health care workers and those in the hostel (8) were used for access to the inmates of the hostel. Table 1 shows the sites from where samples were collected and the number of sites which yielded growth.

Among the 36 samples which were collected from the devices used only by the college faculty and students, 17 (47 \%) yielded growth, of which 14 (82\%) were coagulase negative Staphylococcus spp, 2 (12\%) were Gram positive bacilli and the remaining 1 (6\%) was Gram negative bacilli. Among 16 samples collected from the devices used commonly by both college and hospital side faculty including the turnstiles, 9 (56 \%) yielded growth, 8 (99\%) were Gram positive bacilli and the remaining 1 (1\%) was a Gram negative bacilli. The samples which were collected from the devices used by the health care workers alone were 24. Among them $13(54 \%)$ yielded growth of which 5 (38\%) yielded coagulase negative Staphylococcus spp, 7 (54\%) were Gram positive bacilli and the remaining $1(8 \%)$ was a Gram negative bacilli. The samples which were collected from the devices used by the hostel students were 8 and none of them yielded growth. This is shown in Figure 1.

CONS was isolated from 19 (49\%) of the 39 samples which yielded growth and $3(16 \%)$ 
were of them were methicillin resistant and the rest were methicillin sensitive. The 3 Gram negative bacilli isolated were Enterobacter spp., Acinetobacter spp and
Aeromonas spp. All the 3 isolates were sensitive to all the commonly used antibiotics.

Table.1 Sites of sample collection

\begin{tabular}{|c|l|l|l|}
\hline S.No. & Sample Collection Sites & Number of Samples & Number Yielding Growth \\
\hline 1. & College block & 36 & $17(47 \%)$ \\
\hline 2. & Common places & 16 & $9(56 \%)$ \\
\hline 3. & Hospital block & 24 & $13(54 \%)$ \\
\hline 4. & Hostels & 8 & 0 \\
\hline 5. & Total & $\mathbf{8 4}$ & $\mathbf{3 9}(\mathbf{4 6 \%})$ \\
\hline
\end{tabular}

Fig.1 Distribution of organisms from various devices

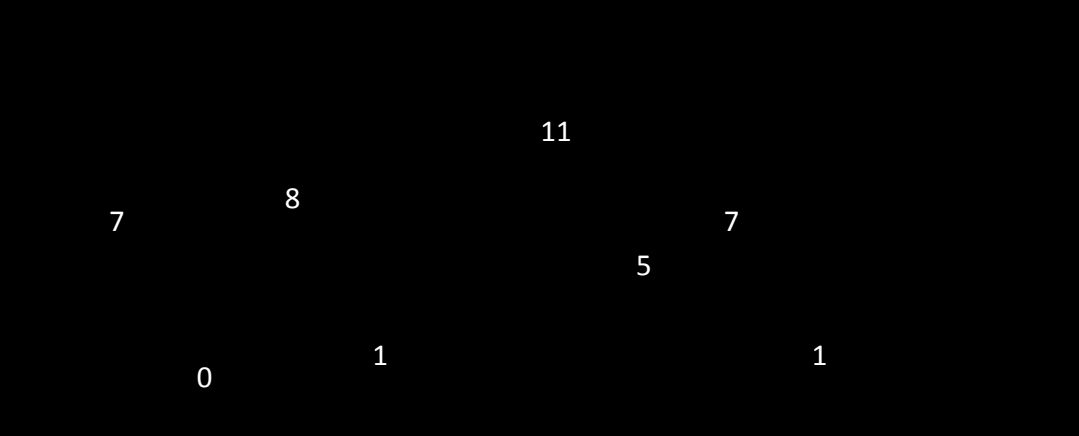

Image.1 Biometric Access Gate

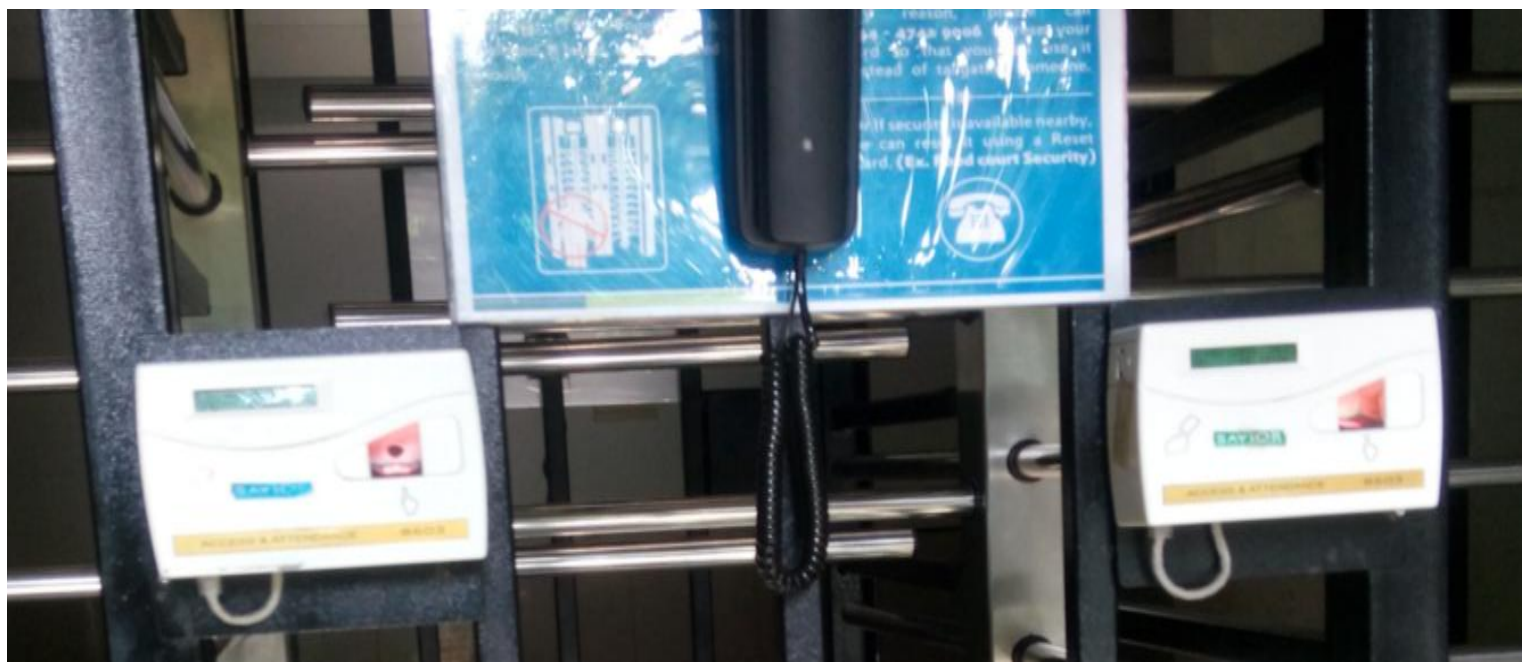




\section{Image.2 Biometric Fingerprinting Device}

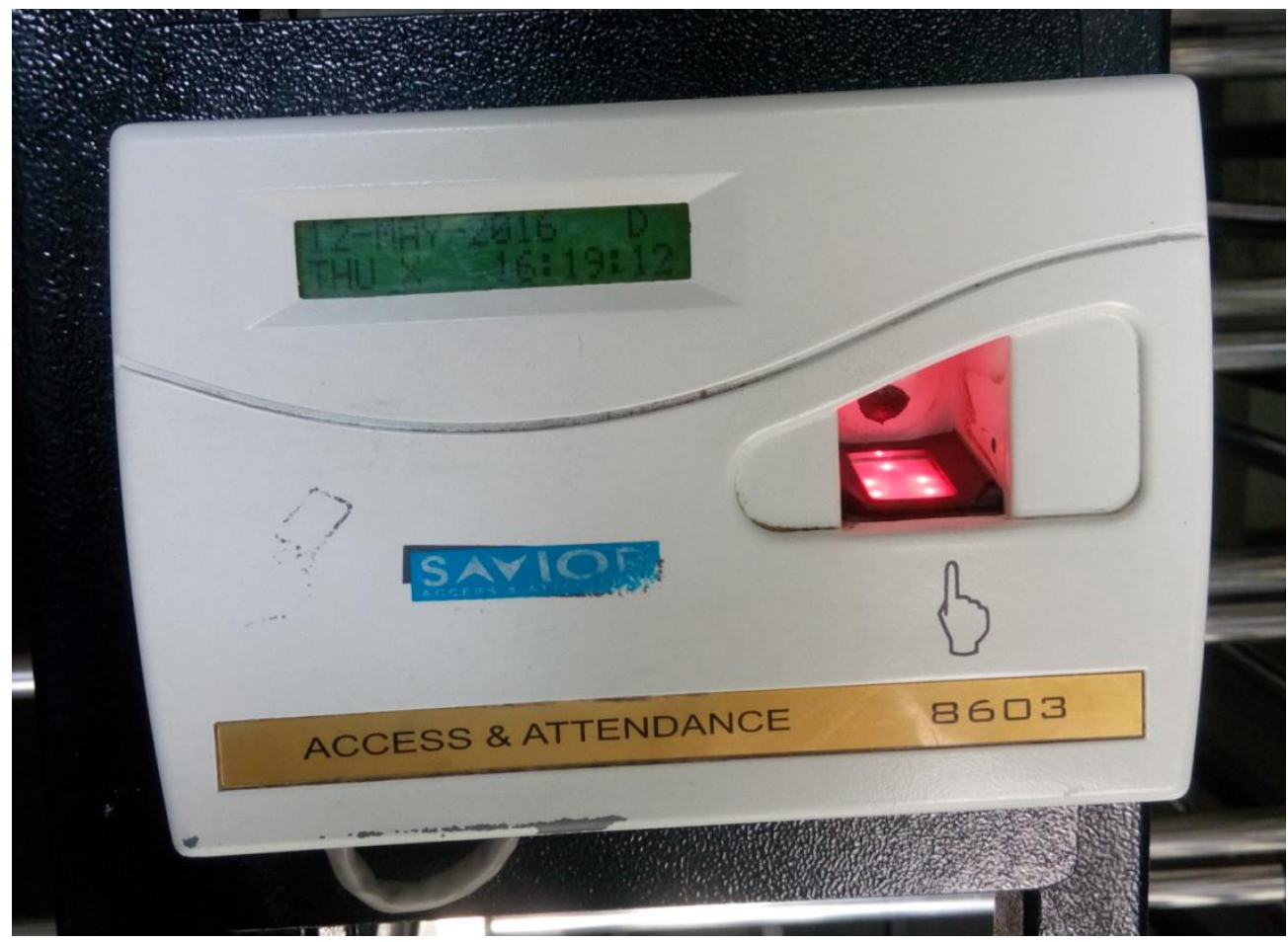

The overall culture positivity rate of all the samples taken from the various biomedical devices was $46 \%$. In a study on the microbial flora on the biometric system in a tertiary care hospital in Madhya Pradesh by Nancy Suhag et al., (2016) the culture positivity was about $33.33 \%$. Christine R. Blomeke et al., (2007) studied the survivability and transferability of these organisms from biometric devices and reported that majority of organisms are transferred in the first 10 minutes after they have been located onto the surfaces of the fingerprinting devices.

Among the 39 of culture positive swabs 49\% were CONS, $43.5 \%$ were Gram positive bacilli and $7.5 \%$ were Gram negative bacilli. Among the 19 isolates of CONS $15 \%$ were methicillin resistant. The Gram negative bacilli isolated were Enterobacter spp., Acinetobacter spp and Aeromonas spp which were sensitive to all the antibiotics. All these Gram negative bacilli have proven roles as nosocomial pathogens. In their study, Nancy Shag et al., isolated only Staphylococcus aureus from the bio-metric devices in their hospital. In a study by Jacobs et al., on bio-metric fingerprinting device in a VISA collecting office Staphylococcus aureus isolation rate was $18.5 \%$ while that of Gram-negative bacteria was $75.1 \%$ (Biometric fingerprinting for visa application, 2008). In another study done by Chigozie J. Uneke et al., on the surface of the Stethoscopes, 53\% of the samples yielded S.aureus, followed by P.aeruginosa (19\%), E.faecalis(14\%) and E.coli (13\%) (Chigozie et al., 2010). A study conducted on the mobile phones of health care personnel in a tertiary care hospital, 65\% were CONS and Staphylococcus aureus was the commonest pathogen to be isolated followed by Gram negative bacilli like E.coli, Pseudomonas sp., Acinetobacter $s p$., etc. A study done by Isaac et al., showed the growth of coagulase-negative Staphylococcus species from 25 keyboards 
(Isaacs et al., 1998). Similar studies conducted on the keyboards in ICU by Bures et al., showed MRSA to be the commonest organism, followed by Enterococcus spp and Enterobacter spp. (Brues et al., 2000) All these findings show that all these devices definitely harbor bacteria on them depending on their location and in their own way serve to transmit them.

In conclusion, the bio-metric finger printing devices are more prone to be transmitting the microorganisms indirectly from one person to another through inanimate objects. In this study $46 \%$ were culture positive. Among 84 swabs only $3(7.5 \%)$ yielded the methicillin resistant coagulase negative Staphylococcus spp and 3 (7.5\%) yielded Gram negative bacilli while the rest were only commensals of the skin namely CONS and Gram positive bacilli. Isolation of few microorganisms from the bio-metric fingerprinting device compared to other studies could be because of sensitization of the health care personnel about the need for hand hygiene and for surface disinfection of high touch areas.

\section{References}

Biometric fingerprinting for visa application. device and procedure are risk factors for infection transmission. J. Travel Med., 15(5): 335-43.

Brues, S., Fishbain, J.T., Uyehara, C.F.T., et al. 2000. Computer keyboards and faucet handles as reservoirs of nosocomial pathogensin the intensive care unit. Am. J. Infect. Control, 8: 465-70.

Carter, M.J. 2005. Enterically infecting viruses: pathogenicity, transmission and significance for food and waterborne infection. J. Appl. Microbiol., 98:1354-1380.
Centers for Disease Control and Prevention. 2002. Guideline for hand hygiene in health-care settings: recommendations of the healthcare infection control practices advisory committee and the HICPAC/SHEA/APIC/IDSA hand hygiene task force. MMWR Recomm Rep., 51: 1-45.

Centers for Disease Control and Prevention. 2003. Guidelines for environmental infection control in health-care facilities: recommendations of CDC and the Healthcare Infection Control Practices Advisory Committee (HICPAC). MMWR Morb. Mortal Wkly. Rep., 52: 1-48.

Chigozie, J., Uneke, et al. 2010. Bacterial contamination of stethoscopes used by health workers: public health implication. J. Infect. Dev. Ctries., 4(7): 436-441.

Christine, R., Bloomeke, Stephen, J., Elliotte, et al. 2007. Bacterial surviablity and transferability on Biometric devices. 1-4244-1129-7/ IEEE. Pg. 80-84.

French, G., Rayner, D., Branson, M., Walsh, M. 1998. Contamination of doctors' and nurses' pens with nosocomial pathogens. Lancet, 351: 213.

Isaacs, D., Daley, A., Dalton, D. 1998. Swabbing computers in search of nosocomial bacteria. Ped. Infect. Dis. J., 17: 533-10.1097/00006454199806000-00025.

Kramer, A., Schwebke, I., Kampf, G. 2006. How long do nosocomial pathogens persist on inanimate surfaces? A systematic review. BMC Infect. Dis., 6: 130.

Nancy Suhag, Arti Jain, Navinchandran, M., Kaore. 2016. Evaluation of Transient Microbial Flora on Biomertic system as a Potential source of Infection in Tertiary Care Hospital, Indian $J$. Microbial. Res., 3(1): 1-4. 
Neely, A.N., Maley, M.P., Warden, G.D. 1999. Computer keyboards as reservoirs for Acinetobacter baumannii in a burn hospital. Clin. Infect. Dis., 29: 1358-1360.

Nirupa, S., Bhabuvignesh, R.N., Jeya, M. 2013. Can mobile phones act as vehicles transmitting Nosocomial infections. Int. J. Pharm. Bio Sci., 859-64.

Scientific Advisory Board of the International Scientific Forum on Home Hygiene. 2006. Hygiene procedures in the home and their effectiveness: a review of the scientific evidence base.

\section{How to cite this article:}

Nirupa, S., V. Gayathri and Priyadarshini Shanmugam. 2016. A Study on Bacterial Flora on the Finger printing Surface of the Biometric Devices at a Tertiary Care Hospital. Int.J.Curr.Microbiol.App.Sci. 5(9): 441-446. doi: http://dx.doi.org/10.20546/ijcmas.2016.509.047 